\section{Crystal structure of 1-(2,4-dimethyl- phenyl)urea}

\author{
L. Jayalakshmi, ${ }^{a}$ C. Ramalingan, ${ }^{a}$ B. Sridhar ${ }^{b}$ and
}

S. Selvanayagam ${ }^{\mathrm{C} *}$

aDepartment of Chemistry, Kalasalingam University, Krishnankoil 626 126, India, baboratory of X-ray Crystallography, Indian Institute of Chemical Technology, Hyderabad 500 067, India, and ${ }^{\mathrm{c}}$ Department of Physics \& International Research Centre, Kalasalingam University, Krishnankoil 626 126, India. *Correspondence

e-mail: s_selvanayagam@rediffmail.com, ramalinganc@gmail.com

Received 11 December 2014; accepted 16 December 2014

Edited by H. Stoeckli-Evans, University of Neuchâtel, Switzerland

In the title urea derivative, $\mathrm{C}_{9} \mathrm{H}_{12} \mathrm{~N}_{2} \mathrm{O}$, the dihedral angle between the benzene ring and the mean plane of the urea group, $\mathrm{N}-\mathrm{C}(=\mathrm{O})-\mathrm{N}$, is $86.6(1)^{\circ}$. In the crystal, the urea $\mathrm{O}$ atom is involved in three $\mathrm{N}-\mathrm{H} \cdots \mathrm{O}$ hydrogen bonds. Molecules are linked via pairs of $\mathrm{N}-\mathrm{H} \cdots \mathrm{O}$ hydrogen bonds, forming inversion dimers with an $R_{2}^{2}(8)$ ring motif. The dimers are linked by further $\mathrm{N}-\mathrm{H} \cdots \mathrm{O}$ hydrogen bonds, forming two-dimensional networks lying parallel to (100).

Keywords: crystal structure; urea; urea derivatives; hydrogen bonding.

CCDC reference: 1039538

\section{Related literature}

For general background to urea derivatives and their biological applications and properties, see: Ramalingan \& Kwak (2008); Ramalingan et al. (2010); Yang et al. (2013); Safari \& Gandomi-Ravandi (2014); Suzuki et al. (2013); Boulahjar et al. (2012); Zhang et al. (2014)

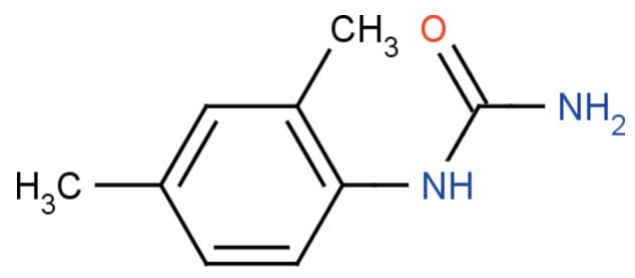

\section{Experimental}

\subsection{Crystal data}

$\mathrm{C}_{9} \mathrm{H}_{12} \mathrm{~N}_{2} \mathrm{O}$

$M_{r}=164.21$

Monoclinic, $P 2_{1} / c$

$a=14.631$ (4) A $b=7.0633(19) \AA$
$c=8.786(2) \AA$
$\beta=93.530(4)^{\circ}$
$V=906.2(4) \AA^{3}$
$Z=4$

\subsection{Data collection}

Bruker SMART APEX CCD areadetector diffractometer 8026 measured reflections

2.3. Refinement

$R\left[F^{2}>2 \sigma\left(F^{2}\right)\right]=0.100$

$w R\left(F^{2}\right)=0.349$

$S=1.59$

1556 reflections

119 parameters

2 restraints $\mu=0.08 \mathrm{~mm}^{-1}$ $T=292 \mathrm{~K}$ $0.20 \times 0.18 \times 0.16 \mathrm{~mm}$
Mo $K \alpha$ radiation

Table 1

Hydrogen-bond geometry $\left(\AA{ }^{\circ}\right)$.

\begin{tabular}{lllll}
\hline$D-\mathrm{H} \cdots A$ & $D-\mathrm{H}$ & $\mathrm{H} \cdots A$ & $D \cdots A$ & $D-\mathrm{H} \cdots A$ \\
\hline $\mathrm{N} 1-\mathrm{H} 1 \cdots \mathrm{O} 1^{\mathrm{i}}$ & 0.86 & 2.23 & $2.941(3)$ & 140 \\
$\mathrm{~N} 2-\mathrm{H} 2 A \cdots \mathrm{O} 1^{\mathrm{i}}$ & $0.86(1)$ & $2.24(2)$ & $2.985(3)$ & $145(3)$ \\
$\mathrm{N} 2-\mathrm{H} 2 B \cdots \mathrm{O} 1^{\mathrm{ii}}$ & $0.86(1)$ & $2.12(1)$ & $2.977(3)$ & $173(4)$ \\
\hline
\end{tabular}

Symmetry codes: (i) $x,-y+\frac{1}{2}, z-\frac{1}{2}$; (ii) $-x+1,-y,-z+1$.

Data collection: SMART (Bruker, 2001); cell refinement: SAINT (Bruker, 2001); data reduction: $S A I N T$; $\operatorname{program}(\mathrm{s})$ used to solve structure: SHELXS97 (Sheldrick, 2008); program(s) used to refine structure: SHELXL2013 (Sheldrick, 2008); molecular graphics: ORTEP-3 for Windows (Farrugia, 2012) and PLATON (Spek, 2009); software used to prepare material for publication: SHELXL2013 and PLATON (Spek, 2009).

\section{Acknowledgements}

CR and SS thank the Vice Chancellor and the management of Kalasalingam University, Krishnankoil, for their support and encouragement.

Supporting information for this paper is available from the IUCr electronic archives (Reference: SU5043).

\section{References}

Boulahjar, R., Ouach, A., Matteo, C., Bourg, S., Ravache, M., le Guével, R., Marionneau, S., Oullier, T., Lozach, O., Meijer, L., Guguen-Guillouzo, C., Lazar, S., Akssira, M., Troin, Y., Guillaumet, G. \& Routier, S. (2012). J. Med. Chem. 55, 9589-9606.

Bruker (2001). SMART and SAINT . Bruker AXS Inc., Madison, Wisconsin, USA.

Farrugia, L. J. (2012). J. Appl. Cryst. 45, 849-854.

Ramalingan, C. \& Kwak, Y. W. (2008). Tetrahedron, 64, 5023-5031.

Ramalingan, C., Park, S. J., Lee, I. S. \& Kwak, Y. W. (2010). Tetrahedron, 66, 2987-2994.

Safari, J. \& Gandomi-Ravandi, S. (2014). J. Mol. Struct. 1074, 71-78.

Sheldrick, G. M. (2008). Acta Cryst. A64, 112-122.

Spek, A. L. (2009). Acta Cryst. D65, 148-155.

Suzuki, H., Utsunomiya, I., Shudo, K., Fukuhara, N., Iwaki, T. \& Yasukata, T. (2013). Eur. J. Med. Chem. 69, 262-277. 


\section{data reports}

Yang, M., Odelberg, S. J., Tong, Z., Li, D. Y. \& Looper, R. E. (2013). Tetrahedron, 69, 5744-5750.
Zhang, D., Debnath, B., Yu, S., Sanchez, T. W., Christ, F., Liu, Y., Debyser, Z., Neamati, N. \& Zhao, G. (2014). Bioorg. Med. Chem. 22, 5446-5453. 


\section{supporting information}

Acta Cryst. (2015). E71, o60-o61 [https://doi.org/10.1107/S2056989014027431]

\section{Crystal structure of 1-(2,4-dimethylphenyl)urea}

\section{Jayalakshmi, C. Ramalingan, B. Sridhar and S. Selvanayagam}

\section{S1. Comment}

Urea and its derivatives are important key starting materials for the construction of biologically important heterocycles (Ramalingan \& Kwak, 2008; Ramalingan et al., 2010; Yang et al., 2013; Safari \& Gandomi-Ravandi, 2014). They display various biological activities viz. antibacterial (Suzuki et al., 2013), antiproliferative and antitumor (Boulahjar et al., 2012), and HIV-1 integrase (Zhang et al., 2014). As a vital reactant and intermediate for the construction of heterocyclic chemical entities of biological importance, the title compound has been synthesized and single crystals were grown by slow evaporation in ethanol.

The single crystal X-ray analysis confirmed the molecular structure, as illustrated in Fig. 1. Methyl carbon atoms, C7 and C8, deviate by -0.000 (1) and -0.040 (1) $\AA$, respectively, from the attached benzene ring. The dihedral angle between benzene ring and the mean plane through the urea atoms $(\mathrm{N} 1 / \mathrm{C} 9 / \mathrm{O} 1 / \mathrm{N} 2)$ is $86.6(1)^{\circ}$.

In the crystal, three strong $\mathrm{N}-\mathrm{H} \cdots \mathrm{O}$ hydrogen bonds stabilize the molecular packing (Fig. 2 and Table 1). Molecules are linked via pairs of $\mathrm{N}-\mathrm{H} \cdots \mathrm{O}$ hydrogen bonds forming inversion dimers with an $\mathrm{R}_{2}^{2}(8)$ ring motif. The dimers are linked by further $\mathrm{N}-\mathrm{H} \cdots \mathrm{O}$ hydrogen bonds forming two-dimensional networks lying parallel to (100); see Table 1 and Fig. 2.

\section{S2. Experimental}

To a solution of 2,6-dimethylaniline $(0.1 \mathrm{~mol})$ in glacial acetic acid $(30 \mathrm{ml})$, was added distilled water $(70 \mathrm{ml})$. Sodium cyanate $(0.1 \mathrm{~mol})$ in medium-hot water $(50 \mathrm{ml})$ was then added in a slow manner with constant stirring. The resulted solution was allowed to stand for $60 \mathrm{~min}$. and then cooled in ice. It was then filtered using a Buchner funnel and the solid obtained was dried using high-vacuum. Single crystals of the title compound were obtained by slow evaporation of a solution in ethanol at room temperature.

\section{S3. Refinement}

Atoms H2A and H2B were located from a difference Fourier map and freely refined. The remaining $\mathrm{H}$ atoms were positioned geometrically and allowed to ride on their parent atoms, with $\mathrm{N}-\mathrm{H}=0.86 \AA$ and $\mathrm{C}-\mathrm{H}=0.93-0.96 \AA$ and with $\mathrm{U}_{\text {iso }}(\mathrm{H})=1.5 \mathrm{U}_{\mathrm{eq}}(\mathrm{C})$ for methyl $\mathrm{H}$ atoms and $=1.2 \mathrm{U}_{\mathrm{eq}}(\mathrm{N}, \mathrm{C})$ for other $\mathrm{H}$ atoms. 


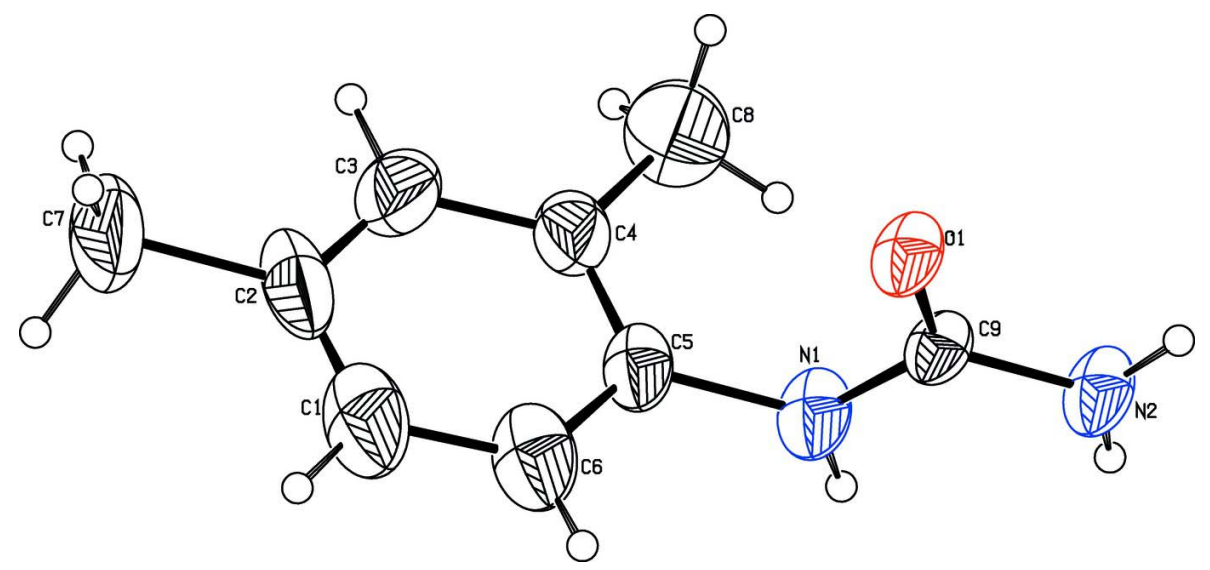

Figure 1

The molecular structure of the title compound, with atom labelling. Displacement ellipsoids are drawn at the 30\% probability level. 


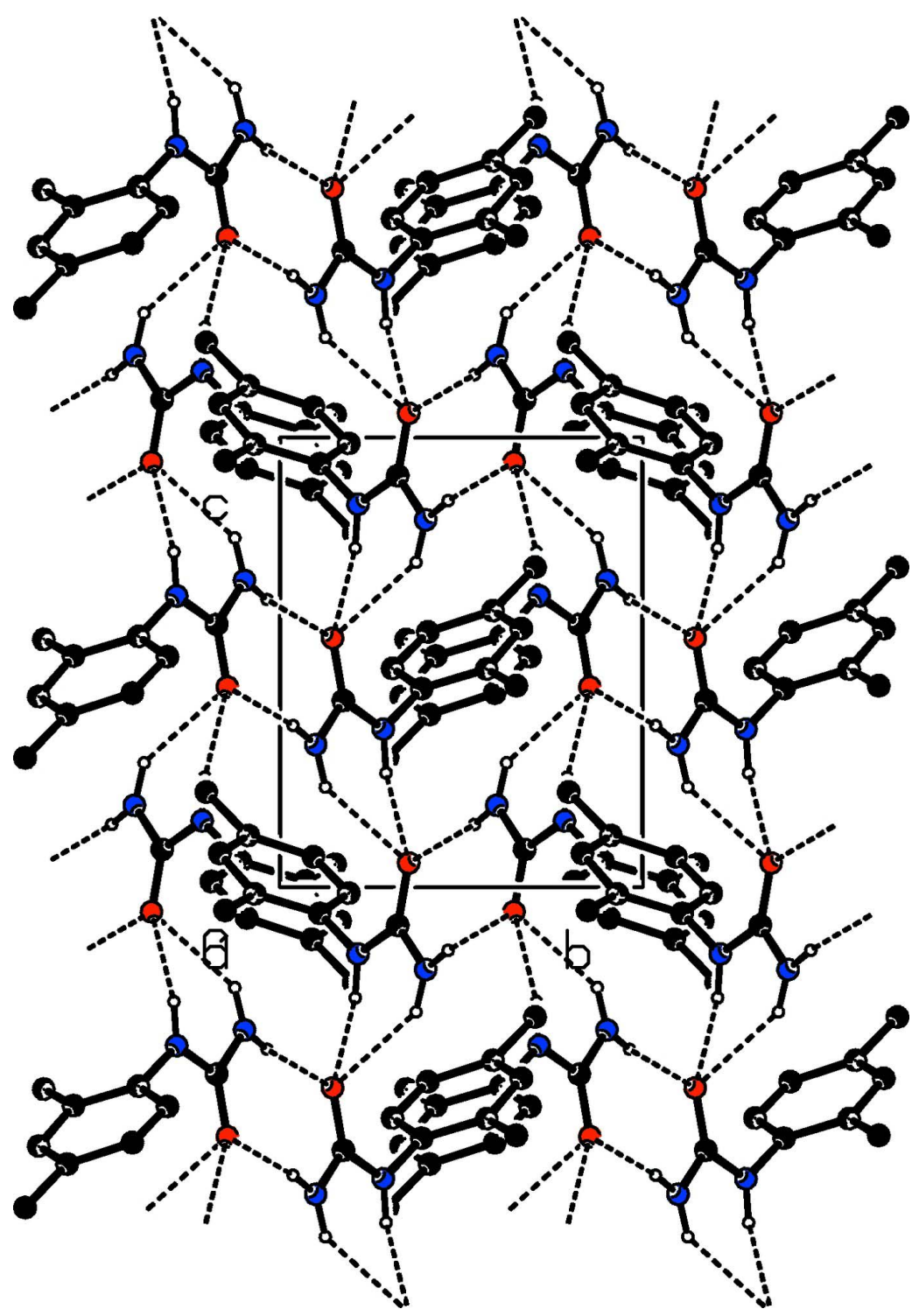

Figure 2

A projection of the crystal packing of the title compound, along the $a$ axis. Hydrogen bonds are shown as dashed lines (see Table 1 for details; $\mathrm{H}$ atoms not involved in hydrogen bonding have been omitted for clarity).

\section{1-(2,4-Dimethylphenyl)urea}

Crystal data

$$
\begin{aligned}
& \mathrm{C}_{9} \mathrm{H}_{12} \mathrm{~N}_{2} \mathrm{O} \\
& M_{r}=164.21 \\
& \text { Monoclinic, } P 2_{1} / c \\
& a=14.631(4) \AA \\
& b=7.0633(19) \AA \\
& c=8.786(2) \AA \\
& \beta=93.530(4)^{\circ} \\
& V=906.2(4) \AA^{3} \\
& Z=4
\end{aligned}
$$

$F(000)=352$

$D_{\mathrm{x}}=1.204 \mathrm{Mg} \mathrm{m}^{-3}$

Mo $K \alpha$ radiation, $\lambda=0.71073 \AA$

Cell parameters from 6568 reflections

$\theta=2.8-24.6^{\circ}$

$\mu=0.08 \mathrm{~mm}^{-1}$

$T=292 \mathrm{~K}$

Block, colourless

$0.20 \times 0.18 \times 0.16 \mathrm{~mm}$ 


\section{Data collection}

Bruker SMART APEX CCD area-detector diffractometer

Radiation source: fine-focus sealed tube $\omega$ scans

8026 measured reflections

1556 independent reflections

\section{Refinement}

Refinement on $F^{2}$

Least-squares matrix: full

$R\left[F^{2}>2 \sigma\left(F^{2}\right)\right]=0.100$

$w R\left(F^{2}\right)=0.349$

$S=1.59$

1556 reflections

119 parameters

2 restraints
1284 reflections with $I>2 \sigma(I)$

$R_{\text {int }}=0.028$

$\theta_{\max }=25.0^{\circ}, \theta_{\min }=2.8^{\circ}$

$h=-17 \rightarrow 17$

$k=-8 \rightarrow 8$

$l=-10 \rightarrow 10$

Hydrogen site location: mixed

$\mathrm{H}$ atoms treated by a mixture of independent and constrained refinement

$w=1 /\left[\sigma^{2}\left(F_{\mathrm{o}}^{2}\right)+(0.2 P)^{2}\right]$

where $P=\left(F_{\mathrm{o}}^{2}+2 F_{\mathrm{c}}^{2}\right) / 3$

$(\Delta / \sigma)_{\max }=0.001$

$\Delta \rho_{\max }=0.87 \mathrm{e}^{-3}$

$\Delta \rho_{\min }=-0.32$ e $\AA^{-3}$

Special details

Geometry. All esds (except the esd in the dihedral angle between two 1.s. planes) are estimated using the full covariance matrix. The cell esds are taken into account individually in the estimation of esds in distances, angles and torsion angles; correlations between esds in cell parameters are only used when they are defined by crystal symmetry. An approximate (isotropic) treatment of cell esds is used for estimating esds involving 1.s. planes.

Fractional atomic coordinates and isotropic or equivalent isotropic displacement parameters $\left(\AA^{2}\right)$

\begin{tabular}{lllll}
\hline & $x$ & $y$ & $z$ & $U_{\text {iso }} * / U_{\text {eq }}$ \\
\hline O1 & $0.40043(14)$ & $0.1453(3)$ & $0.55423(18)$ & $0.0634(8)$ \\
$\mathrm{N} 1$ & $0.32526(19)$ & $0.2851(4)$ & $0.3520(3)$ & $0.0709(10)$ \\
$\mathrm{H} 1$ & 0.3186 & 0.2927 & 0.2543 & $0.085^{*}$ \\
$\mathrm{~N} 2$ & $0.4476(2)$ & $0.0982(4)$ & $0.3174(3)$ & $0.0682(9)$ \\
$\mathrm{H} 2 \mathrm{~A}$ & $0.437(2)$ & $0.125(4)$ & $0.2228(15)$ & $0.060(8)^{*}$ \\
$\mathrm{H} 2 \mathrm{~B}$ & $0.4934(19)$ & $0.036(5)$ & $0.360(4)$ & $0.089(11)^{*}$ \\
$\mathrm{C} 1$ & $0.1253(4)$ & $0.4077(12)$ & $0.5679(5)$ & $0.1144(17)$ \\
$\mathrm{H} 1 \mathrm{~A}$ & 0.0700 & 0.3530 & 0.5912 & $0.137^{*}$ \\
$\mathrm{C} 2$ & $0.1467(3)$ & $0.5877(12)$ & $0.6154(4)$ & $0.122(2)$ \\
$\mathrm{C} 3$ & $0.2259(4)$ & $0.6656(8)$ & $0.5753(5)$ & $0.1049(17)$ \\
$\mathrm{H} 3$ & 0.2404 & 0.7876 & 0.6085 & $0.126^{*}$ \\
$\mathrm{C} 4$ & $0.2873(2)$ & $0.5700(6)$ & $0.4854(4)$ & $0.0794(11)$ \\
$\mathrm{C} 5$ & $0.2652(2)$ & $0.3904(5)$ & $0.4421(3)$ & $0.0666(10)$ \\
$\mathrm{C} 6$ & $0.1852(3)$ & $0.3106(8)$ & $0.4870(4)$ & $0.0921(13)$ \\
$\mathrm{H} 6$ & 0.1721 & 0.1855 & 0.4606 & $0.111^{*}$ \\
$\mathrm{C} 7$ & $0.0822(4)$ & $0.7033(13)$ & $0.7118(7)$ & $0.178(4)$ \\
$\mathrm{H} 7 \mathrm{~A}$ & 0.0806 & 0.6477 & 0.8114 & $0.267^{*}$ \\
$\mathrm{H} 7 \mathrm{~B}$ & 0.0216 & 0.7030 & 0.6630 & $0.267^{*}$ \\
$\mathrm{H} 7 \mathrm{C}$ & 0.1040 & 0.8312 & 0.7212 & $0.267^{*}$ \\
$\mathrm{C} 8$ & $0.3708(4)$ & $0.6517(7)$ & $0.4485(7)$ & $0.1135(16)$ \\
$\mathrm{H} 8 \mathrm{~A}$ & 0.3942 & 0.5853 & 0.3638 & $0.170^{*}$ \\
H8B & 0.4142 & 0.6433 & 0.5348 & $0.170^{*}$ \\
H8C & 0.3612 & 0.7822 & 0.4217 & $0.170^{*}$ \\
& & & &
\end{tabular}




$\begin{array}{lllll}\text { C9 } & 0.39175(19) & 0.1749(4) & 0.4154(3) & 0.0519(8)\end{array}$

Atomic displacement parameters $\left(\AA^{2}\right)$

\begin{tabular}{lllllll}
\hline & $U^{11}$ & $U^{22}$ & $U^{33}$ & $U^{12}$ & $U^{13}$ & $U^{23}$ \\
\hline O1 & $0.0806(14)$ & $0.0787(15)$ & $0.0316(11)$ & $0.0198(9)$ & $0.0094(9)$ & $0.0014(7)$ \\
$\mathrm{N} 1$ & $0.0824(18)$ & $0.0971(19)$ & $0.0338(12)$ & $0.0270(14)$ & $0.0098(11)$ & $0.0074(11)$ \\
$\mathrm{N} 2$ & $0.0857(18)$ & $0.0849(18)$ & $0.0353(14)$ & $0.0251(13)$ & $0.0137(11)$ & $0.0013(10)$ \\
C1 & $0.090(3)$ & $0.193(5)$ & $0.063(2)$ & $0.031(3)$ & $0.025(2)$ & $0.007(3)$ \\
C2 & $0.068(2)$ & $0.245(7)$ & $0.053(2)$ & $0.063(3)$ & $0.0003(17)$ & $-0.015(3)$ \\
C3 & $0.104(3)$ & $0.123(3)$ & $0.084(3)$ & $0.036(3)$ & $-0.019(3)$ & $-0.036(2)$ \\
C4 & $0.0685(19)$ & $0.105(3)$ & $0.064(2)$ & $0.0184(17)$ & $-0.0010(15)$ & $-0.0088(16)$ \\
C5 & $0.0682(19)$ & $0.090(2)$ & $0.0417(16)$ & $0.0245(15)$ & $0.0069(13)$ & $0.0057(13)$ \\
C6 & $0.086(2)$ & $0.128(3)$ & $0.064(2)$ & $0.018(2)$ & $0.0218(17)$ & $0.0214(19)$ \\
C7 & $0.106(4)$ & $0.316(9)$ & $0.112(4)$ & $0.091(5)$ & $-0.002(3)$ & $-0.091(5)$ \\
C8 & $0.112(3)$ & $0.101(3)$ & $0.129(4)$ & $-0.017(3)$ & $0.024(3)$ & $0.004(3)$ \\
C9 & $0.0652(16)$ & $0.0572(15)$ & $0.0342(14)$ & $0.0069(11)$ & $0.0094(11)$ & $-0.0013(9)$ \\
\end{tabular}

Geometric parameters $(\AA, \circ)$

\begin{tabular}{|c|c|c|c|}
\hline $\mathrm{O} 1-\mathrm{C} 9$ & $1.236(3)$ & $\mathrm{C} 3-\mathrm{C} 4$ & $1.406(6)$ \\
\hline $\mathrm{N} 1-\mathrm{C} 9$ & $1.340(4)$ & $\mathrm{C} 3-\mathrm{H} 3$ & 0.9300 \\
\hline $\mathrm{N} 1-\mathrm{C} 5$ & $1.428(4)$ & $\mathrm{C} 4-\mathrm{C} 5$ & $1.358(6)$ \\
\hline $\mathrm{N} 1-\mathrm{H} 1$ & 0.8600 & $\mathrm{C} 4-\mathrm{C} 8$ & $1.407(7)$ \\
\hline $\mathrm{N} 2-\mathrm{C} 9$ & $1.337(4)$ & $\mathrm{C} 5-\mathrm{C} 6$ & $1.377(6)$ \\
\hline $\mathrm{N} 2-\mathrm{H} 2 \mathrm{~A}$ & $0.857(10)$ & $\mathrm{C} 6-\mathrm{H} 6$ & 0.9300 \\
\hline $\mathrm{N} 2-\mathrm{H} 2 \mathrm{~B}$ & $0.863(10)$ & $\mathrm{C} 7-\mathrm{H} 7 \mathrm{~A}$ & 0.9600 \\
\hline $\mathrm{C} 1-\mathrm{C} 6$ & $1.351(7)$ & $\mathrm{C} 7-\mathrm{H} 7 \mathrm{~B}$ & 0.9600 \\
\hline $\mathrm{C} 1-\mathrm{C} 2$ & $1.368(10)$ & $\mathrm{C} 7-\mathrm{H} 7 \mathrm{C}$ & 0.9600 \\
\hline $\mathrm{C} 1-\mathrm{H} 1 \mathrm{~A}$ & 0.9300 & $\mathrm{C} 8-\mathrm{H} 8 \mathrm{~A}$ & 0.9600 \\
\hline $\mathrm{C} 2-\mathrm{C} 3$ & $1.348(9)$ & $\mathrm{C} 8-\mathrm{H} 8 \mathrm{~B}$ & 0.9600 \\
\hline $\mathrm{C} 2-\mathrm{C} 7$ & $1.541(6)$ & $\mathrm{C} 8-\mathrm{H} 8 \mathrm{C}$ & 0.9600 \\
\hline $\mathrm{C} 9-\mathrm{N} 1-\mathrm{C} 5$ & $121.9(2)$ & $\mathrm{C} 6-\mathrm{C} 5-\mathrm{N} 1$ & $120.5(4)$ \\
\hline $\mathrm{C} 9-\mathrm{N} 1-\mathrm{H} 1$ & 119.1 & $\mathrm{C} 1-\mathrm{C} 6-\mathrm{C} 5$ & $122.2(6)$ \\
\hline $\mathrm{C} 5-\mathrm{N} 1-\mathrm{H} 1$ & 119.1 & $\mathrm{C} 1-\mathrm{C} 6-\mathrm{H} 6$ & 118.9 \\
\hline $\mathrm{C} 9-\mathrm{N} 2-\mathrm{H} 2 \mathrm{~A}$ & $117(2)$ & $\mathrm{C} 5-\mathrm{C} 6-\mathrm{H} 6$ & 118.9 \\
\hline $\mathrm{C} 9-\mathrm{N} 2-\mathrm{H} 2 \mathrm{~B}$ & $115(3)$ & $\mathrm{C} 2-\mathrm{C} 7-\mathrm{H} 7 \mathrm{~A}$ & 109.5 \\
\hline $\mathrm{H} 2 \mathrm{~A}-\mathrm{N} 2-\mathrm{H} 2 \mathrm{~B}$ & $128(4)$ & $\mathrm{C} 2-\mathrm{C} 7-\mathrm{H} 7 \mathrm{~B}$ & 109.5 \\
\hline $\mathrm{C} 6-\mathrm{C} 1-\mathrm{C} 2$ & $119.2(6)$ & $\mathrm{H} 7 \mathrm{~A}-\mathrm{C} 7-\mathrm{H} 7 \mathrm{~B}$ & 109.5 \\
\hline $\mathrm{C} 6-\mathrm{C} 1-\mathrm{H} 1 \mathrm{~A}$ & 120.4 & $\mathrm{C} 2-\mathrm{C} 7-\mathrm{H} 7 \mathrm{C}$ & 109.5 \\
\hline $\mathrm{C} 2-\mathrm{C} 1-\mathrm{H} 1 \mathrm{~A}$ & 120.4 & $\mathrm{H} 7 \mathrm{~A}-\mathrm{C} 7-\mathrm{H} 7 \mathrm{C}$ & 109.5 \\
\hline $\mathrm{C} 3-\mathrm{C} 2-\mathrm{C} 1$ & $119.0(4)$ & $\mathrm{H} 7 \mathrm{~B}-\mathrm{C} 7-\mathrm{H} 7 \mathrm{C}$ & 109.5 \\
\hline $\mathrm{C} 3-\mathrm{C} 2-\mathrm{C} 7$ & $119.5(7)$ & $\mathrm{C} 4-\mathrm{C} 8-\mathrm{H} 8 \mathrm{~A}$ & 109.5 \\
\hline $\mathrm{C} 1-\mathrm{C} 2-\mathrm{C} 7$ & $121.5(6)$ & $\mathrm{C} 4-\mathrm{C} 8-\mathrm{H} 8 \mathrm{~B}$ & 109.5 \\
\hline $\mathrm{C} 2-\mathrm{C} 3-\mathrm{C} 4$ & $122.7(5)$ & $\mathrm{H} 8 \mathrm{~A}-\mathrm{C} 8-\mathrm{H} 8 \mathrm{~B}$ & 109.5 \\
\hline $\mathrm{C} 2-\mathrm{C} 3-\mathrm{H} 3$ & 118.7 & $\mathrm{C} 4-\mathrm{C} 8-\mathrm{H} 8 \mathrm{C}$ & 109.5 \\
\hline $\mathrm{C} 4-\mathrm{C} 3-\mathrm{H} 3$ & 118.7 & $\mathrm{H} 8 \mathrm{~A}-\mathrm{C} 8-\mathrm{H} 8 \mathrm{C}$ & 109.5 \\
\hline
\end{tabular}




$\begin{array}{llll}\mathrm{C} 5-\mathrm{C} 4-\mathrm{C} 3 & 117.1(4) & \mathrm{H} 8 \mathrm{~B}-\mathrm{C} 8-\mathrm{H} 8 \mathrm{C} & 109.5 \\ \mathrm{C} 5-\mathrm{C} 4-\mathrm{C} 8 & 121.0(4) & \mathrm{O} 1-\mathrm{C} 9-\mathrm{N} 2 & 122.5(2) \\ \mathrm{C} 3-\mathrm{C} 4-\mathrm{C} 8 & 121.8(4) & \mathrm{O} 1-\mathrm{C} 9-\mathrm{N} 1 & 122.4(2) \\ \mathrm{C} 4-\mathrm{C} 5-\mathrm{C} 6 & 119.7(3) & \mathrm{N} 2-\mathrm{C} 9-\mathrm{N} 1 & 115.1(2) \\ \mathrm{C} 4-\mathrm{C} 5-\mathrm{N} 1 & 119.8(3) & & \\ & & & -2.8(5) \\ \mathrm{C} 6-\mathrm{C} 1-\mathrm{C} 2-\mathrm{C} 3 & 2.3(7) & \mathrm{C} 8-\mathrm{C} 4-\mathrm{C} 5-\mathrm{N} 1 & 90.7(4) \\ \mathrm{C} 6-\mathrm{C} 1-\mathrm{C} 2-\mathrm{C} 7 & -178.3(4) & \mathrm{C} 9-\mathrm{N} 1-\mathrm{C} 5-\mathrm{C} 4 & -88.6(4) \\ \mathrm{C} 1-\mathrm{C} 2-\mathrm{C} 3-\mathrm{C} 4 & 0.5(7) & \mathrm{C} 9-\mathrm{N} 1-\mathrm{C} 5-\mathrm{C} 6 & -4.1(7) \\ \mathrm{C} 7-\mathrm{C} 2-\mathrm{C} 3-\mathrm{C} 4 & -178.9(4) & \mathrm{C} 2-\mathrm{C} 1-\mathrm{C} 6-\mathrm{C} 5 & 2.9(6) \\ \mathrm{C} 2-\mathrm{C} 3-\mathrm{C} 4-\mathrm{C} 5 & -1.6(6) & \mathrm{C} 4-\mathrm{C} 5-\mathrm{C} 6-\mathrm{C} 1 & -177.8(3) \\ \mathrm{C} 2-\mathrm{C} 3-\mathrm{C} 4-\mathrm{C} 8 & -178.1(5) & \mathrm{N} 1-\mathrm{C} 5-\mathrm{C} 6-\mathrm{C} 1 & -174) \\ \mathrm{C} 3-\mathrm{C} 4-\mathrm{C} 5-\mathrm{C} 6 & -0.1(5) & \mathrm{C} 5-\mathrm{N} 1-\mathrm{C} 9-\mathrm{O} 1 & \\ \mathrm{C} 8-\mathrm{C} 4-\mathrm{C} 5-\mathrm{C} 6 & 176.5(4) & \mathrm{C} 5-\mathrm{N} 1-\mathrm{C} 9-\mathrm{N} 2 & \\ \mathrm{C} 3-\mathrm{C} 4-\mathrm{C} 5-\mathrm{N} 1 & -179.4(3) & & \end{array}$

Hydrogen-bond geometry $\left(A,{ }^{\circ}\right)$

\begin{tabular}{lllll}
\hline$D-\mathrm{H} \cdots A$ & $D-\mathrm{H}$ & $\mathrm{H} \cdots A$ & $D \cdots A$ & $D-\mathrm{H} \cdots A$ \\
\hline $\mathrm{N} 1-\mathrm{H} 1 \cdots \mathrm{O} 1^{\mathrm{i}}$ & 0.86 & 2.23 & $2.941(3)$ & 140 \\
$\mathrm{~N} 2-\mathrm{H} 2 A \cdots \mathrm{O} 1^{\mathrm{i}}$ & $0.86(1)$ & $2.24(2)$ & $2.985(3)$ & $145(3)$ \\
$\mathrm{N} 2-\mathrm{H} 2 B \cdots \mathrm{O} 1^{\mathrm{ii}}$ & $0.86(1)$ & $2.12(1)$ & $2.977(3)$ & $173(4)$ \\
\hline
\end{tabular}

Symmetry codes: (i) $x,-y+1 / 2, z-1 / 2$; (ii) $-x+1,-y,-z+1$. 